\title{
Synthesis and Mechanical Characterization of High Density Polyethylene/Graphene Nanocomposites
}

\author{
Abdulaziz S. Alghamdi \\ Mechanical Engineering Department \\ College of Engineering, University of Hail \\ Hail, Saudi Arabia \\ asbg945@hotmail.com
}

\begin{abstract}
The purpose of this work is to investigate the effects of graphene nanosheets (GNSs) addition on the mechanical and thermal properties of high density polyethylene (HDPE). The HDPE/Graphene nanocomposites were synthesized using solution blending approach. HDPE was incorporated with graphene nanosheets in a solvent at various weights of fractions $(0.1,0.2$, 0.4 and $0.5 \mathrm{wt} \%$ ), and then the micro-hardness, elastic modulus, tensile strength, strain at break and thermal properties of the nanocomposites were measured and compared. The results showed that the use of Xylene solvent at high temperature combined with mechanical stirring can fully dissolve HDPE pellets. Scanning electron microscope (SEM) showed that GNSs were homogenously dispersed in the polyethylene matrix at low weights of fractions. The addition of just $0.2 \mathrm{wt} \%$ GNSs resulted in $100 \%$ increase in the micro-hardness value. The elastic modulus and tensile strength properties are proportionally increased with increasing GNSs content up to $0.4 \mathrm{wt} \%$. However, at higher weight of fraction, a reduction in these properties is observed. The crystallinity and strain at break properties are reduced with the addition of GNSs.
\end{abstract}

Keywords-HDPE; polymer; graphene; nanocomposite; thermal; mechanical

\section{INTRODUCTION}

Polymer-based nanocomposites of current academic interest are finding an increasing range of industrial applications, making them the most widely commercialised class of nanocomposites [1]. The incorporation of low weight of fraction of nanoparticles or nanosheets can lead to a significant improvement in the polymer properties like tensile strength, elastic modulus, wear and scratch resistance, electrical and thermal conductivity, thermal and flammability resistance and impact strength [2-5]. In addition, many polymer nanocomposites can be fabricated and processed by the use of methods similar to those used for standard polymers, which is important from an economic point of view. In recent years, researchers have focussed on the synthesis of new nanocomposites, starting from careful material selection and process control either by the direct use of an existing technique or by modified and adapted techniques. Various types of nanofillers have been used in polymer-based nanocomposite fabrication, such as exfoliated clay, modified carbon nanotubes and graphene [6-15]. However, achieving the uniform dispersion of the nanofillers is still an important scientific and technological challenge in nanocomposite fabrication. Graphene nanosheets, with their outstanding thermal, electrical, optical, and mechanical properties as well as their low cost compared to carbon nanotubes can be a cost-effective alternative to carbon nanotubes and can be widely used for the fabrication of polymeric nanocomposites [16-18]. Graphene nanosheets are relatively easier to disperse homogeneously in the neat polymer matrix compared to carbon nanotubes and possess a higher surface area to volume ratio that improves bonding and load transfer properties, and consequently increases the strength [19-21]. High-density polyethylene (HDPE) is a thermoplastic polymer that can be blended with different reinforcement materials to further enhance its overall properties like strength and thermal properties [16, 21, 22]. The mechanical, thermal, electrical and surface hydrophobic properties of HDPE can be improved with the addition of 1 to 8 $\mathrm{wt} \%$ graphene nanoflakes to the matrix using solution blending [16]. However, using large quantities of graphene nanosheets can increase the production cost. Therefore, in this research, small quantities of graphene nanosheets $(0.1-0.5 \mathrm{wt} \%)$ were incorporated with HDPE in a proper solvent, and then the mechanical and thermal properties of the resultant nanocomposites were determined using various techniques.

\section{EXPERIMENTAL WORK}

\section{A. Materials}

The polymer-based nanocomposite presented in this work is high density polyethylene (HDPE) with the addition of graphene nanosheets (GNSs) at various weight percentages (0.1, 0.2, 0.4 and $0.5 \mathrm{wt} \%$ GNSs). HDPE pellets were purchased from SABIC Company, Saudi Arabia in 2016. Graphene nanosheets were purchased from Graphene Laboratories Inc., USA, with average thickness $<3 \mathrm{~nm}$ (between 3-8 graphene monolayers) and lateral dimensions 2-8 microns.

\section{B. Processing}

An in-house solution blending technique was used to perform a well dispersed GNSs into the HDPE matrix. Graphene nanosheets were dispersed in alcohol using sonicator for 30 minutes. Then, the mixture was dried for 24 hours. GNSs were dispersed again in Xylene for 30 min using 
sonication, and then HDPE pellets were added into the dispersions. In order to improve the dissolving of HDPE pellets and the dispersion of GNSs, and also reduce the time needed, hot plate at $130^{\circ} \mathrm{C}$ and mechanical stirrer at 100 r.p.m were applied simultaneously for 2 hours. Then, the dispersions were dried for 24 hours at $150^{\circ} \mathrm{C}$. The nanocomposite material was placed in a square steel mould $(100 \times 100 \times 1 \mathrm{~mm})$ and heated using furnace to $220^{\circ} \mathrm{C}$ for $30 \mathrm{~min}$. Then, the mould was pressed using hydraulic press machine at $7 \mathrm{MPa}$ for $10 \mathrm{~min}$. Water was used to cool the mould to room temperature. The test specimens were cut to the dimensions shown in Figure 1.

\section{Mechanical Testing and Characterization.}

The morphology of the nanocomposites was investigated using a Philips XL30 Environmental Scanning Electron Microscope-Field Emission Gun (ESEM-FEG) from FEI Company (Eindhoven, The Netherlands) and FEI Quanta 250 (USA). DSC, (TA instruments, Shimadzu Dsc60) was used to analyze the effect of GNSs addition on the thermal properties of HDPE. The specimens were sealed in aluminium pans and heated from 35 to $200^{\circ} \mathrm{C}$ at a rate of $10^{\circ} \mathrm{C}$ per minute. The mass fraction of crystallinity is then determined by comparing the heat of fusion with that for fully crystalline polyethylene at the equilibrium melting point $(290 \mathrm{~kJ} / \mathrm{Kg})$. Tensile tests were carried out using an Instron 5969 tensile testing machine from Instron Corporation (Norwood, MA, USA) at room temperature $\left(22 \pm 2^{\circ} \mathrm{C}\right)$. The speed rate used in this work was $1 \mathrm{~mm} / \mathrm{s}$. FLIR camera was used to measure the sample's surface temperature distribution, with temperature range from $-20^{\circ} \mathrm{C}$ to $+150^{\circ} \mathrm{C}$ and accuracy of $\pm 1^{\circ} \mathrm{C}$. All results were plotted using Excel to study the material behavior. Microhardness tests were performed using force of $100 \mathrm{~g}$ for 5 seconds with sharp indenter (Vickers test), the indentation was repeated 5 times and average was taken.

\section{RESULTS AND DISCUSSION}

\section{A. Thermal Analysis}

Figure 2 and Table I present the differential scanning calorimetry results for HDPE and its nanocomposites. It can be seen that the addition of GNSs has no effect on the melting points. However the crystallinity is reduced by $14 \%$ with the addition of $0.1 \mathrm{wt} \%$ graphene. Further reduction is occurred with the increase of graphene percentage. However, more addition of GNSs has no effect on the crystallinity of the nanocomposites.

\section{B. Graphene Nanosheets Dispersion}

The use of Xylene as chemical solvent at high temperature combined with mechanical stirring resulted in $100 \%$ dissolving of HDPE in a short period of time. Usually, to dissolve polyethylene materials, large quantities of solvents are used at room temperature combined with high speed agitation. However, the application of specific temperature can lead to faster dissolving as well as less quantity of solvents. Figure 3 shows the SEM images for the microstructure of HDPE and its nanocomposites. It can be seen that GNSs are dispersed homogenously in the HDPE matrix up to $0.4 \mathrm{wt} \%$ of graphene. However, at $0.5 \mathrm{wt} \%$ graphene, some cavitations and large aggregations of graphene nanosheets can be observed in the microstructure. This can lead to reduction in surface area to volume ratio and consequently reduction of load carrying capacity between graphene and HDPE matrix.

TABLE I. THERMAL PROPERTIES OF HDPE BASED NANOCOMPOSITES

\begin{tabular}{|c|c|c|}
\hline Materials & Crystallinity $\%$ & Melting Point $\left({ }^{\circ} \mathbf{C}\right)$ \\
\hline HDPE & 59.22 & 136.76 \\
\hline HDPE-0.1 wt Graphene & 50.97 & 134.78 \\
\hline HDPE-0.2 wt Graphene & 43.02 & 136.52 \\
\hline HDPE-0.4 wt Graphene & 43.67 & 136.39 \\
\hline HDPE-0.5 wt Graphene & 44.54 & 133.57 \\
\hline
\end{tabular}

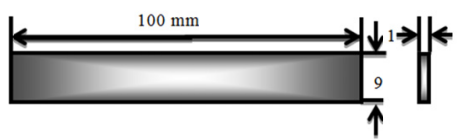

Fig. 1. Dimensions of the specimen

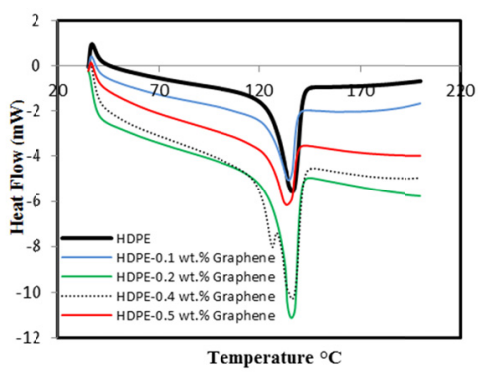

Fig. 2. DSC results for HDPE and its nanocomposites
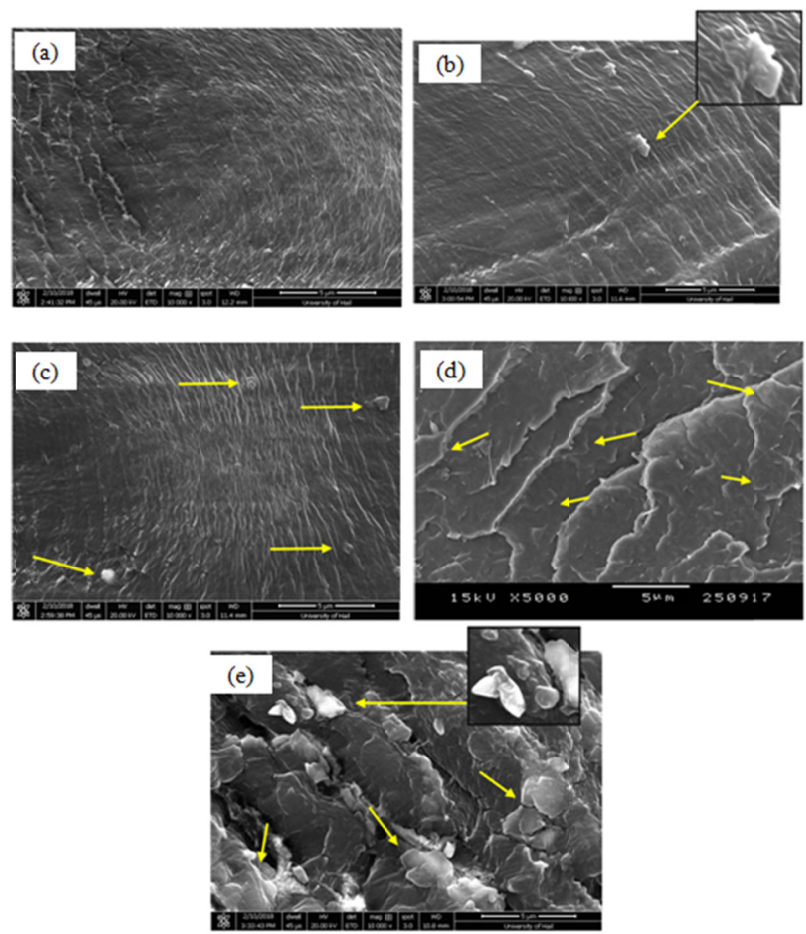

Fig. 3. SEM images for the microstructure of (a) HDPE and (b) HDPE-0.1 wt $\%$ graphene (c) HDPE- $0.2 \mathrm{wt} \%$ graphene (d) HDPE- $0.4 \mathrm{wt} \%$ graphene (e) HDPE- $0.5 \mathrm{wt} \%$ graphene (the arrows indicate the presence of graphene nanosheets or agglomeration) 


\section{Microhardness Results}

It can be seen from Figure 4 that the addition of GNSs to $0.2 \mathrm{wt} \%$ resulted in a significant increase in the micro-hardness values. The embedding of just $0.2 \mathrm{wt} \%$ GNSs into the HDPE matrix almost doubled the micro-hardness values of the nanocomposites comparing to pure HDPE. This can be attributed to the well dispersion of GNSs into the polymer matrix and the good interaction between the nanosheets and the hosting material. Increasing the weight of fraction of GNSs resulted in a reduction in the micro-hardness values and they were almost similar the neat material. This is probably due to the existence of nanoplatelets aggregates and therefore the reduction in the surface to volume ratio.

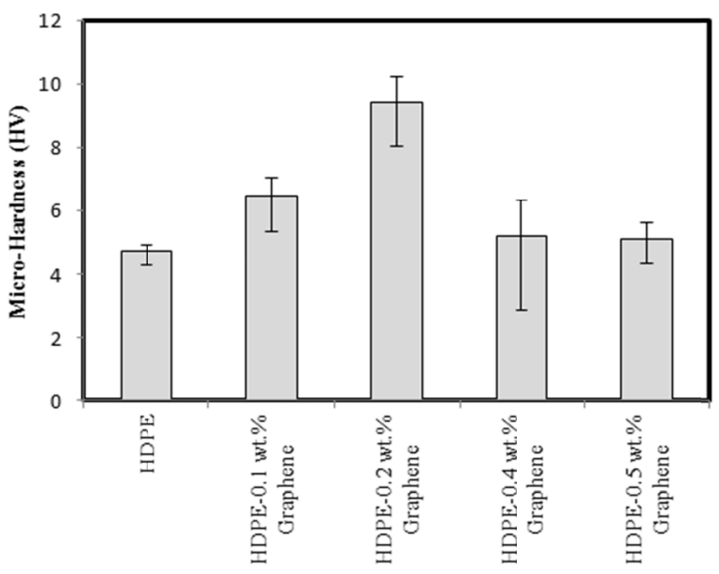

Fig. 4. Effect of GNSs addition on the micro-hardness values.

\section{Tensile Test Results}

During the tensile testing, the phenomena of heat generation during plastic deformation can potentially affect the mechanical properties of polymeric materials. Several researches $[6,7,10]$ provided evidence that the existence of nanofillers in the polyethylene matrix can increase the heat generation during plastic deformation. Therefore the heat is measured to avoid such influences. It is found that the maximum temperature generated during the plastic deformation reached $35^{\circ} \mathrm{C}$, which has no significant effects on the material properties. Therefore, the effect of heat generation during plastic deformation is neglected in this work. Figure 5 shows the effect of GNSs addition on the percentage of strain at break. It can be seen that the presence of these nanofillers caused significant reduction in the strain at break. The embedding of $0.2 \mathrm{wt} \%$ of GNSs into the HDPE matrix resulted in 30\% reduction in the strain at brake. More reduction up to $60 \%$ occurs with the addition of $0.5 \mathrm{wt} \%$ GNSs. On the other hand, the elastic modulus and tensile strength of the HDPE are increased with increase of the weight percentage of GNSs up to $0.4 \mathrm{wt} \%$. At $0.5 \mathrm{wt} \%$ GNSs, a reduction is observed in both elastic modulus and tensile strength values as seen in Figure 5. This is probably due to the presence of GNSs agglomerations, which affected the surface area to volume ration and consequently the stress distribution during the application of the tensile load.

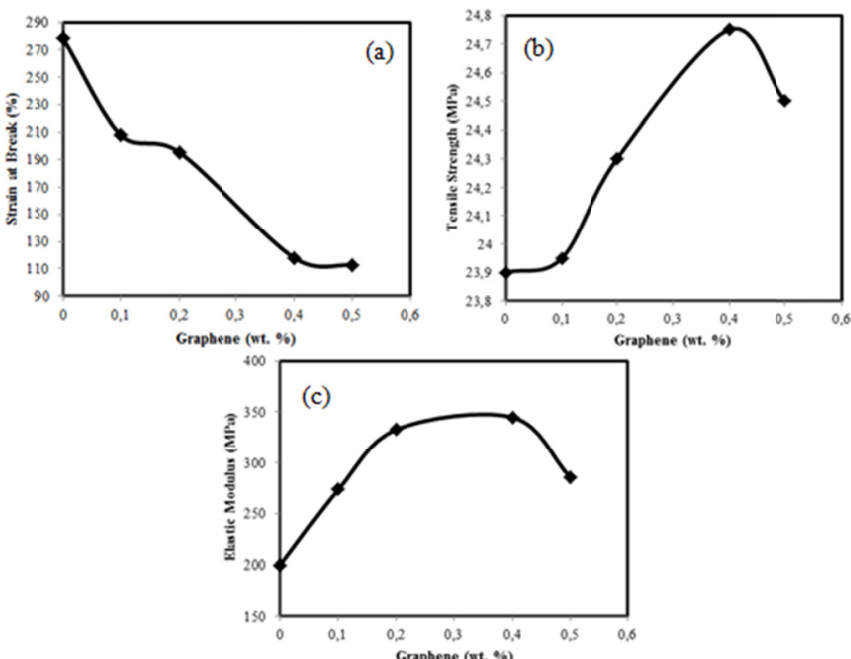

Fig. 5. Effect of GNSs addition (a) on strain at brake, (b) on tensile strength, (c) on the elastic modulus

\section{CONCLUSION}

The main findings in this work are summarised as:

1) The use of solution blending approach at high temperature combined with mechanical stirring resulted in fully HDPE pellets dissolving in a short period compared to the use of this method at room temperature.

2) The addition of low weight fraction of GNSs resulted in a remarkable increase in the microhardness values, which reached double that of HDPE with the addition of only $0.2 \mathrm{wt} \%$ GNSs.

3) The heat generation during plastic deformation was measured and the effect was ignored.

4) The addition of GNSs caused significant reduction in the percentage of strain at break.

5) Elastic modulus and tensile strength are increased with the addition of reinforcements, however at higher weight fraction $(0.5 \mathrm{wt} \%)$, a reduction in these properties is observed.

\section{REFERENCES}

[1] R. Bogue, "Nanocomposites: a review of technology and applications", Assembly Automation, Vol. 31, No. 2, pp. 106-112, 2011

[2] M. Alexandre, P. Dubois, T. Sun, J. M. Garces, R. Jerome, "Polyethylene layered silicate prepared by polymerisation-filling techniques: synthesis and mechanical properties", Polymer, Vol. 43, No. 8, pp. 2123-2132, 2002

[3] S. S. Ray, M. Okamoto, "Polymer/layered silicate nanocomposites: a review from preparation to processing", Progress in Polymer Science, Vol. 28, No. 11, pp. 1539-1641, 2003

[4] K. Yusoh, J. Jin, M. Song, "Subsurface mechanical properties of polyurethane/organoclay nanocomposite thin films studied by nanoindentation", Progress in Organic Coatings, Vol. 67, No. 2, pp. 220224,2010

[5] Z. Z. Wang, P. Gua, Z. Zhang, "Indentation and scratch behavior of nano-SiO2/polycarbonate composite coating at the micro/nano-scale", Wear, Vol. 269, No. 1-2, pp. 21-25, 2010

[6] A. S. Alghamdi, I. A. Ashcroft, M. Song, D. Cai, "Morphology and strain rate effects on heat generation during the plastic deformation of polyethylene/carbon black nanocomposites", Polymer Testing, Vol. 32, No. 6, pp. 1105-1113, 2013 
[7] A. S. Alghamdi, I. A. Ashcroft, M. Song, D. Cai, "Nanoparticle type effects on heat generation during the plastic deformation of polyethylene nanocomposites", Polymer Testing, Vol. 32, No. 8, pp. 1-9, 2013

[8] A. S. Alghamdi, I. A. Ashcroft, M. Song, "Creep Resistance of novel polyethylene/carbon black nanocomposites", International Journal of Materials Science and Engineering, Vol. 2, No. 1, pp. 1-5, 2014

[9] A. S. Alghamdi, I. A. Ashcroft, M. Song. "High Temperature Effects on the Nanoindentation Behaviour of Polyethylene-based Nanocomposites", International Journal of Computational Methods and Experimental Measurements, Vol. 3, No. 2, pp. 79-88, 2015

[10] C. V. Gorwade, A. S. Alghamdi, I. A. Ashcroft, V. V. Silberschmidt, M. Song, "Finite element analysis of the high strain rate testing of polymeric materials", Modern Practice in Stress and Vibration Analysis, Journal of Physics: Conference Series, Vol. 382, Art. No. 012043, pp. 16,2012

[11] A. S. Alghamdi, "Nanoparticle type effects on the scratch resistance of polyethylene-based nanocomposites", International Journal of Advanced and Applied Sciences, Vol. 4, No. 4, pp. 1-6, 2017

[12] D. R. Paul, L. M. Robeson "Polymer nanotechnology: nanocomposites", Polymer, Vol. 49, No. 15, pp. 3187-3204, 2008

[13] M. Rahmat, P. Hubert, "Carbon nanotube-polymer interactions in nanocomposites: a review", Composites Science and Technology, Vol. 72, No. 1, pp. 72-84, 2011

[14] X. Jiang, L. T. Drzal, "Multifunctional high density polyethylene nanocomposites produced by incorporation of exfoliated graphene nanoplatelets: crystallization, thermal and electrical properties", Polymer Composites, Vol. 33, No. 4, pp. 636-642, 2012

[15] M. M. Shokrieh, M. R. Hosseinkhani, M. R. Naimi-Jamal, H. Tourani, "Nanoindentation and nanoscratch investigations on graphene-based Nanocomposites", Polymer Testing, Vol. 32, No. 1, pp. 45-51, 2013

[16] R. Asmatulu, W. S. Khan, R. J. Reddy, M. Ceylan, "Synthesis and analysis of injection-molded nanocomposites of recycled high-density polyethylene incorporated with graphene nanoflakes", Polymer Composites, Vol. 36, No. 9, pp. 1565-1573, 2015

[17] D. Verma, P. C. Gope, A. Shandilya, A. Gupta, "Mechanical-thermal electrical and morphological properties of graphene reinforced polymer composites: a review", Transactions of the Indian Institute of Metals, Vol. 67, No. 6, pp. 803-816, 2014

[18] X. Jiang, L. T. Drzal, "Multifunctional high-density polyethylene nanocomposites produced by incorporation of exfoliated graphene nanoplatelets 2: crystallization, thermal and electrical properties", Polymer Composites, Vol. 33, No. 4, pp. 636-642, 2012

[19] R. J. Reddy, R. Asmatulu, W. S. Khan, "Electrical properties of recycled plastic nanocomposites produced by injection molding", ASME International Mechanical Engineering Congress and Exposition, Vancouver, Canada, Vol. 5, pp. 1321-1327, 2010

[20] R. Asmatulu, W. S. Khan, R. J. Reddy, M. Ceylan, H. E. Misak, "Physical properties of injection molded graphene nanocomposites from recycled plastics", SAMPE Fall Technical Confernece, Fort Worth, USA, Vol. 11, pp. 17-20, 2011

[21] R. J. Reddy, Preparation, characterization and properties of injection molded graphene nanocomposites, MSc Thesis, Wichita State University, 2010

[22] S. N. Tripathi, G. S. S. Rao, A. B. Mathur, R. Jasra, "Polyolefin/graphene nanocomposites: a review", RSC Advances, Vol. 7, pp. 23615-23632, 2017 